\title{
Neurorehabilitation in basilar artery occlusion in a young patient with thrombophilia and antiphospholipid antibody syndrome - a case report
}

\author{
Maria-Gabriela Catană ${ }^{\mathbf{1}}$, Corina Roman-Filip ${ }^{1,2}$
}

Corresponding author: Corina Roman-Filip, E-mail: corinaromanf@yahoo.com

\begin{abstract}
Twenty percent of strokes occurring in young patients are represented by posterior ischemic strokes. Acute basilar occlusion is a devastating, life-threatening condition, with the highest mortality in young patients. We present the case of a 33-year-old female patient, without vascular risk factors or oral contraceptive treatment, admitted to our department through the emergency ward for comatose state - Glasgow Coma Scale (GCS) 11 points, headaches, right-sided hemiparesis, dizziness and vomiting, with acute onset. CT angiography was performed, which showed left vertebral artery with no flow in the intradural section and absent flow in the basilar artery. After more than 12 hours from onset, endarterectomy was excluded; initiation of treatment with heparin 1000 IU/hour was decided. MRI performed after 24 hours revealed: subacute median and left paramedian pontine ischemic stroke, subacute stroke in the base of the left midbrain peduncle. The following diagnosis was established: pontine ischemic stroke caused by two autoimmune diseases: thrombophilia and antiphospholipid antibody syndrome. Our patient started rehabilitation very early and was discharged with the following neurological sequelae: tetraparesis with the predominance of left hemiparesis: $4 / 5$ on the Medical Research Council strength scale (MRC) - right limbs, $3 / 5$ on the Medical Research Council strength scale (MRC) - left limbs, and dysphagia for liquids.
\end{abstract}

Key words: ischemic stroke, young patient, neurorehabilitation,

\section{Introduction}

Twenty percent of strokes occurring in young patients are represented by posterior ischemic strokes. Acute basilar occlusion is a devastating, life-threatening condition, with the highest mortality in young patients (1). Studies on the outcome of basilar artery occlusion using a conventional treatment approach have been small and have described the outcome of highly selected patients or used very broad definitions, including vertebral and branch artery occlusions, finding a case fatality of $86 \%$ (2). Basilar artery occlusion represents $1 \%$ of all strokes and currently, there is no consensus on the best treatment strategy for these patients. While endovascular reperfusion therapy has been demonstrated to improve outcomes in anterior circulation stroke, its benefit in acute basilar artery occlusion (BAO) has not been confirmed in randomized controlled trials.

Antiphospholipid antibody syndrome is a rare cause of stroke in young patients, especially when associated with thrombophilia or other hypercoagulable states. These autoantibodies appear to cause clinical symptoms by working in concert with phospholipid-binding proteins, which subsequently bind to endothelial and other cells leading to a pro-inflammatory or hypercoagulable state. Stroke associated with antiphospholipid antibodies occurs most commonly in young people and should be considered as a possible cause in these groups (3).

If there is an association between inherited thrombophilias and arterial stroke, then it is a weak one, probably enhanced by other prothrombotic risk factors, such as antiphospholipid antibody syndrome or systemic lupus erythematosus (SLE) (4).

Ischemia may transiently induce antiphospholipid antibodies, and prospective studies examining stroke incidence among patients have found that the presence of lupus anticoagulant poses a greater risk than other antiphospholipid antibodies. Consistent associations between young ischemic stroke and the presence of lupus anticoagulant and anticardiolipin antibodies are seen, inflammation, especially IL-6, being highlighted as a risk factor in the development of stroke (5). 


\section{Case report}

We present the case of a 33-year-old female patient, with neither vascular risk factors nor oral contraceptive treatment, admitted to our department through the emergency ward for comatose state CGS 11 points, headaches, right-sided hemiparesis, dizziness and vomiting, with acute onset. At admission, the patient had a blood pressure value of $120 / 80 \mathrm{mmHg}$ and a rhythmic heart rate, $100 \mathrm{~b} / \mathrm{min}$. She had no fever and no other abnormalities at the general examination. Neurological examination revealed: divergent strabismus of the left eye, rightsided hemiplegia, 0/5 MRC, diminished right osteotendinous reflexes, and right plantar extension. Our patient was not able to maintain her gait. In the emergency service, cerebral CT was performed which was not conclusive, showing no ischemic lesion. Blood tests revealed only mild leukocytosis. Due to the fact that the patient's condition worsened tetraplegia with skew eye deviation, decerebration rigidity and a comatose state - GCS 4 points, CT angiography was performed. This showed left vertebral artery with no flow in the intradural section and absent flow in the basilar artery (Figure 1). After more than 12 hours from onset, endarterectomy was excluded; initiation of treatment with heparin 1000 IU/hour was decided. MRI performed in the Radiology Department after 24 hours revealed: subacute median and left paramedian pontine ischemic stroke, subacute stroke in the base of the left midbrain peduncle (Figures 2, 3). Differential diagnosis with other autoimmune disorders such as multiple sclerosis or Balo-like lesions and neuromyelitis optica (NMO) was discussed. In the case of Balo-like lesions, the lesions are characteristic, with rings of demyelination, surrounded by partially demyelinated regions, reflecting concentricity within the lesion (6), and even though brainstem involvement, with or without transverse myelitis, is rarely seen in the classic type of NMO, this diagnosis was also ruled out by MRI which showed an ischemic pontine lesion, knowing that the most characteristic brainstem lesion in NMO involves the area postrema (7).

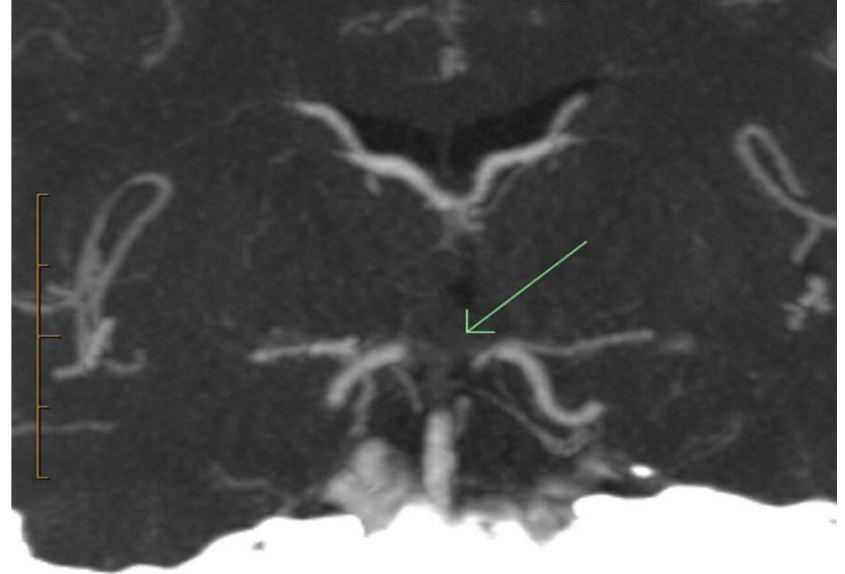

Fig. 1. CT angiography - Basilar occlusion

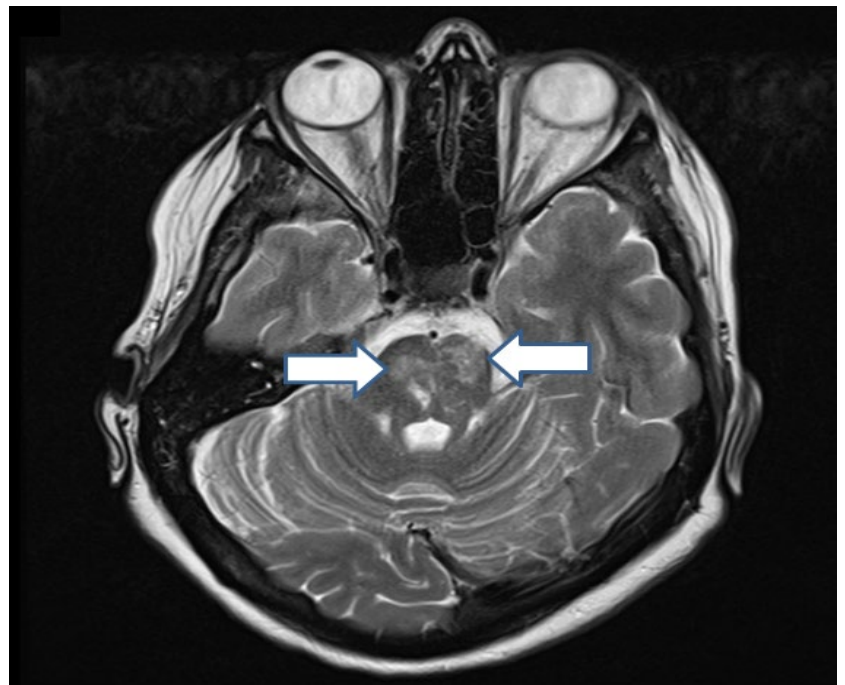

Fig. 2. T2 - FLAIR MRI - Subacute median and left paramedian pontine ischemic stroke

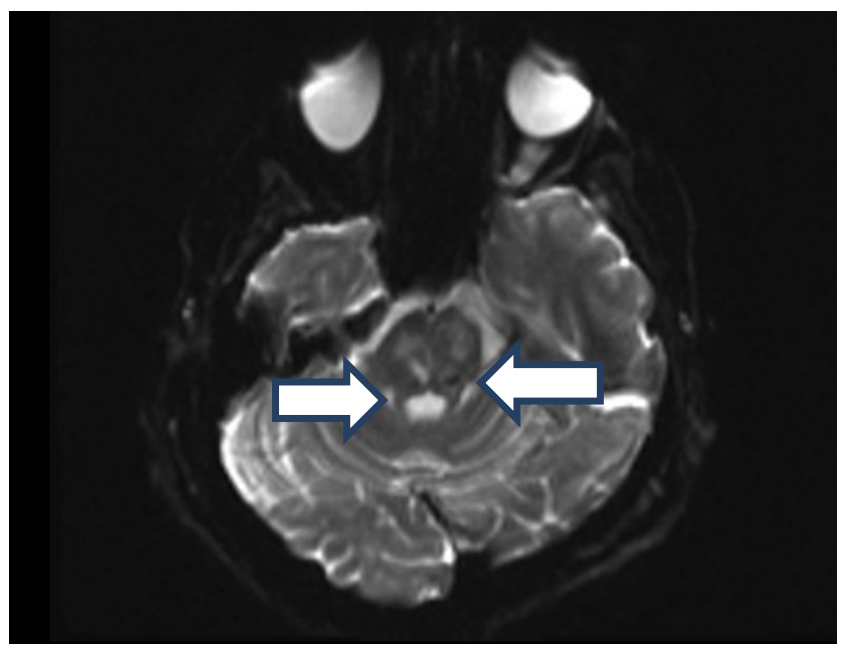

Fig. 3. DWI MRI - Pontine ischemic stroke 
Based on all the information gathered, a hematological consultation was considered advisable. The hematologist recommended blood tests for different autoimmune disorders: thrombophilia, antiphospholipid antibody syndrome and lupus anticoagulant. For thrombophilia, the following were positive: factor VIII (heterozygous genotype), MTHFR C677T (homozygous genotype), and PAI1 (heterozygous genotype). Antiphospholipid syndrome and lupus anticoagulant antibodies were also positive. When deglutition was possible, dicumarin therapy was initiated. Due to the fact that the patient was in good condition, it was decided to start rehabilitation as soon as possible. Recent studies have shown that the greatest degree of recovery occurred relatively rapidly during the first 4 weeks of treatment (i.e. neurological impairments); recovery was also observed within 3 to 6 months of stroke, but to a lesser extent (8).

Rehabilitation was started early and the results did not take long to appear. Studies suggest that if a patient with a certain level of cognitive ability can acquire functional independence, one should focus more on treatment to maximize the recovery of impairment during the early post-stroke period. Our patient started rehabilitation very early: simple motor exercises and task-specific training, motor training being oriented towards achieving the goals relevant to the functional needs of the patient. Motor rehabilitation was accompanied by speech and swallowing therapy. It is known that the duration of rehabilitation therapy is not clearly established: for stroke survivors, rehabilitation should provide as much scheduled therapy (occupational therapy and physiotherapy) as possible, with a minimum of three hours a day (9). After 14 days, the patient was included in a rehabilitation program consisting of daily physical exercises, for 3 hours per day, performed in the Rehabilitation Department of our hospital. After the rehabilitation program, she was discharged with the following neurological sequelae: tetraparesis with the predominance of left hemiparesis: 4/5 MRC - right limbs, 3/5 MRC - left limbs, and dysphagia for liquids, with a Barthel Index score of 11 (at admission, the Barthel Index score was 0 ).

\section{Discussions}

In as many as $35 \%$ of cases, the underlying etiology of stroke in young adults is still unclear. While atherosclerosis remains an important risk factor (accounting for 15-25\% of strokes in young adults), cardioembolic stroke is more common among younger patients $(15-35 \%$ of cases). Other causes that are more frequent in young people include extracranial artery dissection (2-25\% of cases), migraine (up to $20 \%$ of cases), and drug use (up to $5 \%$ of cases, depending on the frequency of use in a given population).

Oral contraceptive use has been implicated in up to $8 \%$ of young stroke cases in some populations (10). Apart from antiphospholipid antibody syndrome (5$10 \%$ of cases), inherited coagulation disorders do not appear to play an important role in young stroke in the absence of right-to-left venoarterial shunting (10). Cerebral venous thrombosis is an uncommon cause of young stroke $-1 \%$; usually, the risk of CVT is considered high in women older than 35 years who use oral contraception for a long period of time (10, 11). Despite all this being known, the etiology of $30 \%$ of all strokes remains unknown - the ESUS concept.

One of the ESUS criteria is excluding a major-risk cardioembolic source of embolism, which in our case was ruled out by the fact that our patient was monitored in the intensive care unit for 24 hours/day. Post-acute care and rehabilitation are often considered a costly area of care to be trimmed, but without recognition of their clinical impact and ability to reduce the risk of downstream medical morbidity resulting from immobility, depression, loss of autonomy, and reduced functional independence. The provision of comprehensive rehabilitation programs with adequate resources, dose, and duration is an essential aspect of stroke care and should be a priority in these redesign efforts (5).

The particularity of our case is that our patient was diagnosed with two autoimmune diseases that caused the procoagulant status, whereas each of them separately could not cause such extensive cerebral ischemia. It is known that thrombophilia by itself cannot induce a procoagulant state; another risk factor (for example, another autoimmune disease, smoking, contraceptive pills, etc.) should be present.

\section{Conclusions}

The presented case fits into the stroke statistics described by the literature, in which thrombophilia is rarely the etiology of arterial ischemia. Most often, thrombophilia is known to be the etiology of cerebral venous thrombosis. In addition, the association between thrombophilia and antiphospholipid antibody syndrome is known to raise thrombotic risk. 
An improvement in lower motor function is observed in about $65 \%$ of patients with initial motor deficits. The rate of clinical recovery is relatively rapid during the first few weeks after a stroke, but then slows considerably between 1 and 3 months later. Between 3 and 6 months after stroke, recovery slows so much as to be barely noticeable, although there appears to be an overall trend toward some additional recovery during this time (6).

\section{Conflict of interest}

There is no conflict of interest for any of the authors regarding this paper.

\section{Informed consent}

An informed consent was obtained from the patient included in this study.

\section{References}

1. Lee Y, Yoon W, Kim SK, Baek B, Kim G, Kim J, Park M. Acute Basilar Artery Occlusion: Differences in Characteristics and Outcomes after Endovascular Therapy between Patients with and without Underlying Severe Atherosclerotic Stenosis. AJNR Am J Neuroradiol. 2017 Aug;38(8):1600-1604.

2. Schonewille W, Algra A, Serena J, Molina C. Outcome in patients with basilar artery occlusion treated conventionally. J Neurol Neurosurg Psychiatry 2005;76:1238-1241.

3. Topel C, Brey R. Antiphospholipid Antibody Syndrome. Primer on Cerebrovascular Disease. 2017, pp. 590-594, doi: 10.1016/B978-0-12803058-5.00116-8.

4. Morris J, Singh S, Fisher M. Testing for Inherited Thrombophilias in Arterial Stroke. Can It Cause More Harm Than Good? Stroke. 2010 Dec; 41(12):2985-90.

5. Griffiths D, Sturm J. Epidemiology and Etiology of Young Stroke. Hindawi Stroke Research and Treatment. 2011:209370.

6. Roman-Filip C, Ungureanu A, Prăvariu I. Balólike lesion associated with psoriasis and chronic autoimmune thyroiditis. Acta Neurologica Belgica. 2015; vol. 115( 4): 793-796.

7. Roman-Filip C, Ungureanu A, Cernuşcă-Miţariu M. Painful tonic spasms and brainstem involvement in a patient with neuromyelitis optica spectrum disorder. Polish Journal of Neurology and Neurosurgery. 2016;50(1):55-8.

8. Bo Lee K, Lim S, Hoon Kim K, Jeon Kim K, Kim Y, Chang W, Yeom J, Kim Y, Hwang B. Six-month functional recovery of stroke patients: a multi-time-point study. International Journal of Rehabilitation Research. 2015 Jun; 38(2): 173180.

9. Stanescu I, Dogaru G, Bulboaca A, Stan A, Stanca D, Blesneag A, Kallo R. Combined pharmacological and motor training interventions for recovery of upper limb function in subacute ischemic stroke. Balneo Research Journal.2017; 8(3): 114-120.

10. Roman Filip C, Rociu C, Beldean L. Cerebral venous sinus thrombosis in a patient with polycystic ovary syndrome. Acta Endocrinologica. 2010; 6(1), 10.4183/aeb.2010.123.

11. Winstein C, Stein J, Arena R, Bates B, Cherney L, Cramer S, Deruyter F, Eng J, Fisher B. A Guideline for Healthcare Professionals from the American Heart Association/American Stroke Association, Stroke. 2016 Jun; 47(6):e98-e169. 